\title{
紧带边流形的整齐嵌入和内浸 ${ }^{*}$
}

\author{
郭 景 美 \\ (南开大学数学系, 天津 300071)
}

\section{摘要}

本文研究了 $n$ 维紧带边流形到圆盘 $D^{n+k}$ 的整齐嵌入和内浸问题 ${ }^{[1-6]}$. 给出了在 亚稳定范围内整齐嵌入和内浸的充分性条件及整齐嵌入的整齐同痕类集合和内浸正 则同伦类集合的表示形式。

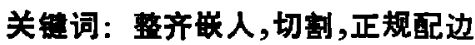

\section{一、预备 知 识}

设 $M$ 为 $n$ 维带边流形, $D^{n+k}$ 表示 $(n+k)$ 维圆盘, $f: M \rightarrow D^{n+k}$ 是嵌人, 如 $f(\partial M) \subset$ $\partial D^{n+k}=s^{n+k-1}$, 且对任 $x \in \partial M, f$ 的切映射 $T f$ 满足 $T_{x} f\left(T_{x} M\right) \not \subset T_{f(x)} S^{n+k-1}$, 则我们称 $f$ 为 $M$ 到 $D^{n+k}$ 的整齐㙂入, 记作 $M \bigcap^{n} D^{n+k}$. 设 $N E_{m b}\left(M, D^{n+k}\right)$ 表示 $M$ 到 $D^{n+k}$ 的整齐 筑人的整齐同痕类集合, $\operatorname{Im}\left(M, D^{n+k}\right)$ 表示 $M$ 到 $D^{n+k}$ 内浸正则同伦类集合.

引理 1. 设 $M$ 是一个 $n$ 维带边流形, $M \bigcap^{n} D^{n+k}(k>2), t: S^{\prime} \times D^{n-r} \rightarrow$ int $M \times 1$ 是嵌 人, 令 $W=M \times I \cup_{1+\left(s^{r} \times D^{n-r}\right)} D^{r+1} \times D^{n-r}$, 如 $2 r \leqslant n \leqslant 2 k-3$, 则 $M$ 在 $D^{n+k} \times O$ 中的 整齐㛶入可扩张为 $W$ 到 $D^{n+k} \times l$ 的整齐嵌人, 且满足 $W \cap D^{n+k} \times 1=\overline{M-f\left(S^{r} \times D^{n-r}\right)}$ - $U_{i f\left(s^{r}\right.} \times s^{n-r-1} ; D^{r+1} \times S^{n-r-1}, W \cap\left(S^{n+k-1} \times I\right)=\partial M \times I$. 如 $2 r \leqslant n<2 k-3$, 则 $W$ 的 扩张就同痕而言是唯一的.

证. 令 $\nu\left(M, D^{n+k}\right)$ 表示 $M$ 在 $D^{n+k}$ 中整齐畹入的法丛, 令 $C=D^{n+k}-\operatorname{int} D(\nu(M$, $\left.\left.D^{n+k}\right)\right)$, 容易看出 $C$ 是 $(k-2)$-连通的, 以下证明过程和文献 [1]中第一节中的切割嵌人引! 理证明完全类似,只需把 $S^{n+k}$ 换成 $D^{n+k}$, 我们就不证了.

引理 2. 设 $(W, F, B)$ 是正规配边(定义参看文献 [71), 这里 $W$ 是连系两个 $n$ 维闭流形 $N$ 和 $M$ 的配边， $F: W \rightarrow M \times I$ 是连续映射且满足 $\left.F\right|_{M: M \rightarrow M \times 1}$ 是恒等映射, $\xi^{k}$ 是 $M \times I$ 上的 $k$ 维向量丛， $k \gg n ， v^{k}$ 是 $W$ 在 $S^{n+k} \times I$ 中整齐资入的稳定法丛, $B: v^{k} \rightarrow \xi^{k}$ 是 覆盖 $F$ 的从映射, 则存在连系 $N$ 和 $M$ 的正规配边 $\left(W^{\prime}, F^{\prime}, B^{\prime}\right)$, 满足 $W^{\prime}$ 是由 $N \times I$ 通过贴 附一列指数 $\leqslant\left[\frac{n}{2}\right]+1$ 的手柄而得到( 㐾义看义献 $\left[8^{\circ}\right.$ ).

证. 由于 $\left.F\right|_{\mathrm{N}}: N \rightarrow M$ 为正规映射(定义参看文献[7]), 因此由文献[8]中的 (10.1) $\mathrm{A}$ 及 
Poincare 对偶性, 由 $N \times I$ 可通过贴附一系列指数 $\leqslant\left[\frac{n}{2}\right]+1$ 的手柄，切割成正规配边 $\left(W_{1}\right.$,

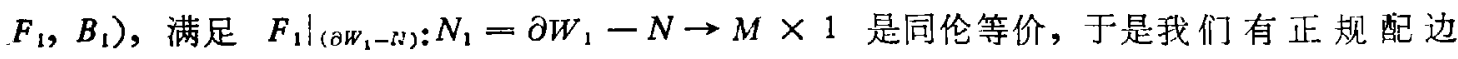
. $\left(W_{1} \cup_{I N} W, F_{1} \cup F, B_{1} \cup B\right)$, 这里 $F_{1} \cup F: W_{1} \cup_{1 N} W \rightarrow M \times I \cup_{1} M_{\times 1=1 M^{* 0}} M \times I=M \times I$

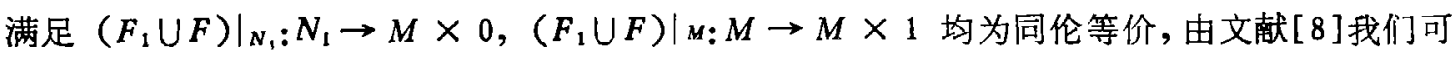
在 $W_{1} \cup_{I N} W$ 的内部进行切割使 $F_{1} \cup F$ 变成 $\left[\frac{n+1}{2}\right]$-等价. 设通过这些切割, $\left(W_{1} \cup_{I N} W\right.$, $\left.F_{1} \cup F, B_{1} \cup B\right)$ 正规配边于 $\left(W_{2}, F_{2}, B_{2}\right)$, 由于 $F_{2}$ 为 $\left[\frac{n+1}{2}\right]$-等价, 易知 $N_{1} \subset W_{2}, M \subset$ $W_{2}$ 满足在 $i \leqslant\left[\frac{n+1}{2}\right]-1$ 时, 导出 $i$ 维同伦群同构, 因此 $W_{2}$ 可看作由 $N_{1} \times I$ 通过贴附 指数为 $\left[\frac{n}{2}\right],\left[\frac{n+1}{2}\right]$ 的手柄而得到. 令 $W^{\prime}=W_{1} \cup_{N_{1}} W_{2}, F^{\prime}=F_{1} \cup F_{2}, B^{\prime}=B_{1} \cup B_{2}$, 则 $W^{\prime}$ 是 $N$ 到 $M$ 的配边, 且 $W^{\prime}$ 可看作对 $N \times I$ 贴附一系列指数 $\leqslant\left[\frac{n}{2}\right]+1$ 手柄而得到, 显 然 $\left(W^{\prime}, F^{\prime}, B^{\prime}\right)$ 是所要正规配边.

引理 3. 设 $(W, F, B)$ 是正规配边, 这里 $W$ 是 $n$ 维带边流形 $N$ 到 $n$ 维带边流形 $M$ 的配边 且 $\partial N=\partial M$, 映射 $F: W \rightarrow M \times 1$ 满足 $F_{(M \cup \partial M \times I)}$ 为恒等映射, $\xi^{k}$ 为 $M \times I$ 上的 $k$ 维向 量丛 $(k \gg n), \nu^{k}$ 是 $W$ 在 $D^{n+k} \times I$ 中整齐嵌人的稳定法丛， $B: \nu^{k} \rightarrow \xi^{k}$ 是 $F$ 上的丛映射， 则存在 $N$ 到 $M$ 的正规配边 $\left(W^{\prime}, F^{\prime}, B^{\prime}\right)$ 满足 $W^{\prime}$ 是由 $N$ 的内部对 $N \times I$ 贴附一系列指数 $\leqslant$ $\left[\frac{n}{2}\right]+1$ 的手柄而得到.

证. 类似引理 2 , 只是在切割过程中需保持边界不动,根据所给条件及文献 [8]中定理 5.6 和 6.4 , 这是可以办到的.

命面. 设 $(W, F, B)$ 是正规配边, 这里 $W$ 是连系两个 $n$ 维带边流形 $N$ 和 $M$ 的配边, $\partial W=N \cup P \cup M, \partial P=\partial N \cup \partial M$; 映射 $F: W \rightarrow M \times I$ 满足 $\left.F\right|_{M}$ 为恒等映射,此外设 $\xi^{k}$ 为 $M \times I$ 上的 $k$ 维向量丛 $(k \gg n), \omega^{k}$ 是 $W$ 整齐嵌入在 $D^{n+k} \times I$ 中的稳定法丛, $B: \omega^{k} \rightarrow$ $\xi^{k}$ 是 $F$ 上的丛映射. 则存在连系 $N$ 和 $M$ 的配边 $W^{*}$ ，满足 $W^{*}$ 是先由 $N$ 的边界 $\partial N$ 贴附一 系列指数 $\leqslant\left[\frac{n-1}{2}\right]+1$ 的手柄, 然后再由内部贴附一系列指数 $\leqslant\left[\frac{n}{2}\right]+1$ 手柄而得到.

证. 类似引理 2 .

\section{二、主要定理}

设 $M$ 为 $n$ 维带边流形, 对于任 $q>n+1$, 知 $M$ 可整齐嵌入 $D^{n+k+q}$, 设脱入法丛为 $\nu^{k+q}$, $\nu_{\partial M}^{k+q}=\nu_{i \partial M}^{k+q}$. 把 $M$ 在 $D^{n+k+q}$ 中管壮邻域的余撮合成一点，我们可得到一个映射 $C_{M} \in$ $\pi_{n+k+q}\left(T \nu_{M}^{k+q}, T \nu_{\partial M}^{k+q}\right)$, 这里 $T \nu_{M}^{k+q}, T \nu_{\partial M}^{k+q}$ 分别是相应丛的 Thom 空间. 由于 $\pi_{n+k+q}$ - $\left(T \nu_{M}^{k+q}, T \nu_{\partial M}^{k+q}\right) \cong \pi_{n+k}\left(Q^{q} T \nu_{M}^{k+q}, Q^{q} T \nu_{\partial M}^{k+q}\right)$, 从而 $C_{M}$ 可看作 $\pi_{n+k}\left(Q^{q} T \nu_{M}^{k+q}, Q^{q} T \nu_{\partial M}^{k+q}\right)$ 中 一个元素.

设 $V_{k+q, q}(M)$ 为 $v_{M}^{k+q}$ 所伴生的纤维为 $V_{k+q, q}$ 的丛, $\pi_{q}$ 为丛投影. 令 $V_{k+q, q}(\partial M)=$ $V_{k+q, q}(M)_{1 a^{M}}$. 对于任 $x \in V_{k+q, q}(M)$, 则 $x$ 是 $R^{n+k+q}$ 中某 $k+q$ 维超平面 $R^{k+q}$ 的一个 二维正交标架,设 $\tilde{x}$ 表示 $x$ 所张成的子空间在 $R^{k+q}$ 中的正交补,于是我们有映射 


$$
\begin{aligned}
\tilde{\nu}_{k+q}: V_{k+q, q}(M) & \rightarrow B O(k) . \\
x & \mapsto \tilde{x}
\end{aligned}
$$

设 $\nu_{k+q}: M \rightarrow B O(k+q)$ 表示 $\nu_{M}^{+q}$ 的分类映射； $i: B O(k) \rightarrow B O(k+q)$ 表示自然创 含,于是我们有可换图:

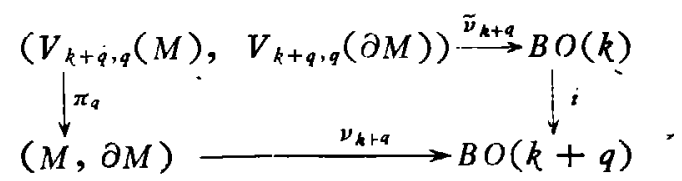

由(2.1)式,显然我们有以下丛映射:

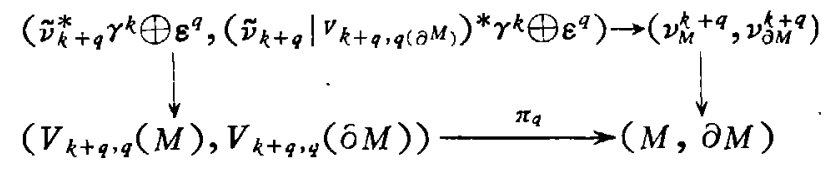

这里 $\gamma^{k}$ 是 $k$ 维泛丛.

由(2.2)式,显然我们可导出下面的 Thom 复形间的映射:

$$
t\left(\pi_{q}\right):\left(\Sigma^{q} T \tilde{\nu}_{k+q}^{*} \gamma^{k}, \Sigma^{q} T\left(\tilde{\nu}_{k+q} \mid v_{k+q, q(\partial M)}\right)^{*} \gamma^{k}\right) \rightarrow\left(T \nu_{M}^{k+q}, T \nu_{\partial M}^{k+q}\right),
$$

令 $V^{k}(M)=\lim _{q \rightarrow \infty} V_{k+q \cdot q}(M), \tilde{\nu}=\lim _{q \rightarrow \infty} \tilde{\nu}_{k+q}, \quad \nu=\lim _{q \rightarrow \infty} \nu_{k+q}, \pi=\lim _{q \rightarrow \infty} \pi_{q}$, 则(2.1)式变为

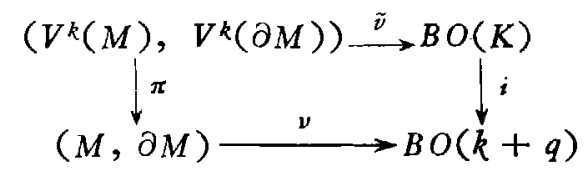

令 $I=\lim _{q \rightarrow \infty} \Omega^{q} t\left(\pi_{q}\right), \tilde{\nu}^{*} \gamma^{k}=V^{k}\left(\nu_{M}\right),\left(\tilde{\nu} \mid V^{k}(\partial M)\right)^{*} \gamma^{k}=V^{k}\left(\nu_{\partial} M\right)$, 由（2.3）式, 可导出映 射

$$
I:\left(Q^{\infty} \Sigma^{\infty} T V^{k}\left(\nu_{M}\right), Q^{\infty} \Sigma^{\infty} T V^{k}\left(\nu_{\partial M}\right)\right) \rightarrow\left(Q^{\infty} T \nu_{M}^{\infty}+k, Q^{\infty} T \nu_{\partial}^{\infty}{ }^{+k}\right)
$$

令

$$
\begin{gathered}
l_{q}:\left(T \tilde{\nu}_{k+q}^{*} \gamma^{k}, T\left(\tilde{\nu}_{k+q} l^{\prime l_{k+q}, q}(\partial M)\right)^{*} \gamma^{k}\right) \rightarrow \\
\left(Q^{q} \Sigma^{q} T \tilde{\nu}_{k+q}^{*} \gamma^{k}, Q^{q} \Sigma^{q} T\left(\tilde{\nu}_{k+q} \mid v_{k+q, q}(\partial M)\right)^{*} \gamma^{k}\right)
\end{gathered}
$$

表示 $\left(\Sigma^{q} T \tilde{\nu}_{k+q}^{*} \gamma^{k}, \Sigma^{q} T\left(\tilde{\nu}_{k+q} \mid v_{k+q, q^{(\partial M)}}\right)^{*} \gamma^{k}\right)$ 到本身恒等映射所诱导出来的映射,记 $l=\lim _{q \rightarrow 0} l_{g}$, 则我们有映射 $l:\left(T V^{k}\left(\nu_{M}\right), T V^{k}\left(\nu_{\partial M}\right)\right) \rightarrow\left(Q^{\infty} \Sigma^{\infty} T V^{k}\left(\nu_{M}\right), Q^{\infty} \Sigma^{\infty} T V^{k}\left(\nu_{\partial M}\right)\right)$, 从而我们有下 图:

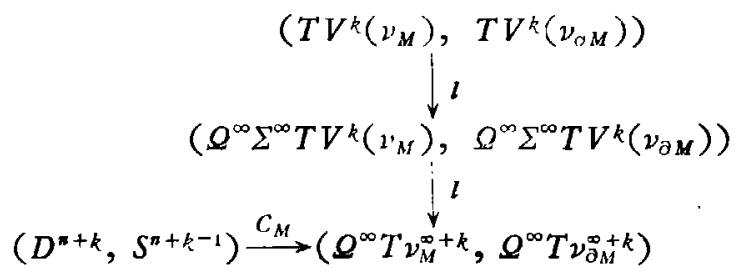

.令 $E=I \circ l$, 则我们的主要结果如下:

主要定理. 设 $M$ 为 $n$ 维带边流形, $\partial M$ 为一个连通分支, 如 $n>4, k>2$, 则存在映射 $\phi: N E_{m} b\left(M, \partial M ; D^{n+k}, S^{n+k-1}\right) \rightarrow E_{*}^{-1}\left(C_{M}\right) \subset \pi_{n+k}\left(T V^{k}\left(\nu_{M}\right), T V^{k}\left(\nu_{\partial M}\right)\right)$ $\theta: \operatorname{Im}\left(M, D^{n+k}\right) \rightarrow I_{*}^{-1}\left(C_{M}\right) \subset \pi_{n+k}\left(Q^{\infty} \Sigma^{\infty} T V^{k}\left(\nu_{M}\right), Q^{\infty} \Sigma^{\infty} T V^{k}\left(\nu_{\partial M}\right)\right)$ 
满足如果 $2 k \geqslant n+3$, 则 $\phi$ 是满的, 如 $2 k>n+3$, 则 $\phi$ 是 $1-1$ 的; 如 $2 k \geqslant n+1$, 则 $\theta$ 是 满的, 如 $2 k>n+1$, 则 $\theta$ 是 1-1 的.

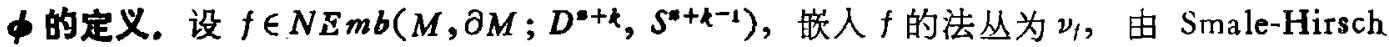
定理,知丛

$$
\left(V_{k}(M), V_{k}(\partial M) \stackrel{\pi}{\longrightarrow}(M, \partial M)\right.
$$

存在截口 $S_{f}$, 因此存在丛映射

及 Thom 复形之间的映射:

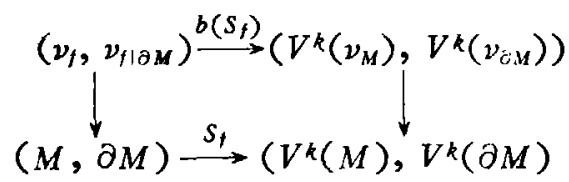

$$
\operatorname{tb}\left(S_{f}\right):\left(T \nu_{f}, T\left(\nu_{f \mid \partial M}\right)\right) \rightarrow\left(T V^{k}\left(\nu_{M}\right), T V^{k}\left(\nu_{\partial M}\right)\right),
$$

把嵌人 $t$ 的管状邻域的余撮合成一点, 于是我们有映射

$$
C_{f:}\left(D^{n+k}, S^{n+k-1}\right) \rightarrow\left(T \nu_{f}, T\left(\nu_{/ \mid \partial M}\right)\right) .
$$

由(2.5)式中 $C_{M}, E$ 的定义及 $\mathrm{tb}\left(S_{f}\right)$ 的定义, 显然 $\mathrm{tb}\left(S_{f}\right)_{*}\left[C_{f}\right] \in E_{*}^{-1}\left(C_{M}\right)$, 我们就定义 $\phi([f])=\mathrm{tb}\left(S_{f}\right)_{*}\left[C_{f}\right]$.

$\theta$ 的定义. 设 $f \in \operatorname{Im}\left(M, D^{n+k}\right)$, 由 Smale-Hirsch 定理, 则存在类似 $\phi$ 定义中的 $b\left(S_{f}\right)$ 和 $\mathrm{tb}\left(S_{t}\right)$, 且对于充分大的 $q$, 组合映射

$$
M \stackrel{f}{\longrightarrow} D^{n+k} \stackrel{C}{\longrightarrow} D^{n+k+q}
$$

正则同伦于一个整齐嵌人 $f_{1}:(M, \partial M) \rightarrow\left(D^{n+k+q}, S^{n+k+q-1}\right)$, 于是我们有撮合映射

$$
C_{f_{1}}:\left(D^{n+k+q}, S^{n+k+q-1}\right) \rightarrow\left(T \nu_{f_{1}}, T \nu_{f_{1} \mid \partial M}\right) \text {. }
$$

注意 $\left(D^{n+k+q}, S^{n+k+q-1}\right)=\left(\Sigma^{q} D^{n+k}, \Sigma^{q} S^{n+k-1}\right),\left(T \nu_{f_{1}}, T \nu_{f_{1} \mid \partial M}\right)=\left(\Sigma^{q} T \nu_{f}, \Sigma^{q} T \nu_{f \mid \partial M}\right)$, 又由 于 $q$ 充分大,从而有 $C_{f_{1}} \in \pi_{n+k+q}\left(T \nu_{f_{1}}, T \nu_{f_{1} \mid \partial M}\right)=\pi_{n+k+q}\left(\Sigma^{q} T \nu_{i}, \Sigma^{q} T \nu_{f \mid \partial M}\right) \cong \pi_{n+k}\left(Q^{\infty} \Sigma^{\infty} T \nu_{f}\right.$, $\left.Q^{\infty} \Sigma^{\infty} T v_{f \mid \partial M}\right)$, 设在上同构中 [ $C_{f_{1}}$ ] 对应 [ $\left.\widetilde{C}_{f}\right]$, 由 (2.5) 式中 $I$ 的定义及 $\operatorname{tb}\left(S_{j}\right)$ 的定义, 显 然 $\left(\Omega^{\infty} \Sigma^{\infty} \mathrm{tb}\left(S_{f}\right)\right)_{*}\left[\widetilde{C}_{f}\right] \in I_{*}^{-1}\left(C_{M}\right)$, 我们就定义 $\theta([f])=\left(\Omega^{\infty} \Sigma^{\infty} \mathrm{tb}\left(S_{f}\right)\right)_{*}\left[\widetilde{C}_{f}\right]$.

\section{三、主要定理的证明}

$2 k \geqslant n+3$ 时, $\phi$ 是满的. 对任意 $d \in \pi_{n+k}\left(T V^{k}\left(\nu_{M}\right), T V^{k}\left(\nu_{\partial M}\right)\right)$, 由于 $T V^{k}\left(\nu_{M}\right)$ 减 去一点是一个流形，而 $V^{k}(M)$ 是它的余维数为 $k$ 的整齐子流形，因此我们可以选 $d$ 的与 $V^{k}(M)$ 横截的某一表示元, 仍记为 $d$, 于是 $d^{-1}\left(V^{k}(M)\right)=N$ 是 $D^{n+k}$ 中一个 $n$ 维整齐带 边子流形. 设 $N$ 在 $D^{a+k}$ 中法丛为 $v\left(N, D^{n+k}\right)$, 于是有丛映射

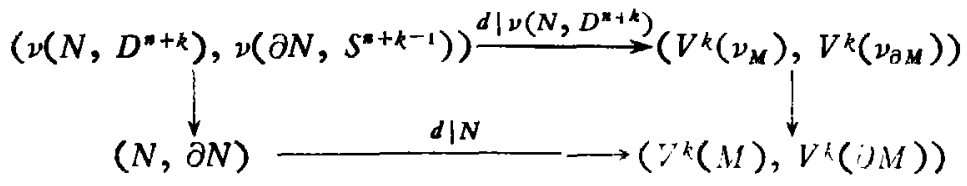

由上面的丛映射可导出下面的丛映射：

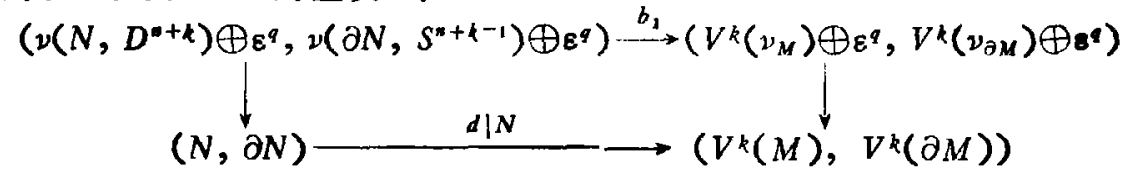


由(2.4)式,存在从胦射

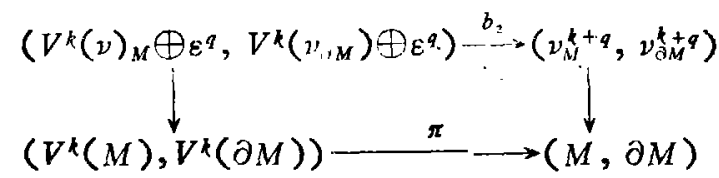

因此 $\left(\pi \circ d_{\mid N}, b_{2} \circ b_{1}\right)$ 是丛映射, 由于. $d \in E_{*}^{-1}\left(C_{M}\right)$, 从而由文献[8]中引理 $10.1_{\mathrm{B}}$, 知 $\pi \circ d_{\mid N}$ 正 规配边于一个平凡的正规映射, 即存在正规配边 $\left(W^{n+1}, F, B\right)$, 这里 $W$ 是一个 $n+1$ 维带 边流形, $\partial W=N \cup P \cup M, \partial P=\partial N \cup \partial M, F$ 是 $\pi \circ d_{1 N} \cup i d M$ 的扩张, $B$ 是 $F$ 上的丛映射且 是 $b_{2} \circ b_{1} \cup i d \nu_{M}^{k+q}$ 的扩张.

下面我们证明 $N$ 在 $D^{n+k}$ 中的整齐嵌入可扩充为一个 $W$ 在 $D^{n+k} \times I$ 中的整齐嵌入 $G$, 且满足 $G^{-1}\left(D^{n+k} \times 1\right)=M, G^{-1}\left(D^{n+k} \times 0\right)=N, G^{-1}\left(S^{n+k-1} \times I\right)=P$.

由于 $N$ 和 $M$ 的配边 $W$ 是正规配边, 由前面的命题, 我们可设 $W$ 是通过先在 $\partial N$ 上贴附一 系列指数 $\leqslant\left[\frac{n-1}{2}\right]+1$ 的手柄, 然后在内部贴附一系列指数 $\leqslant\left[\frac{n}{2}\right]+1$ 手柄而得到.

由于 $n \leqslant 2 k-3$, 从而更有 $n-1 \leqslant 2 k-3$, 因此由文献[1]中切割族入引理, 则存在 $P$ 到 $S^{n+k-1} \times I$ 的嵌入 $g_{1}$, 满足 $g_{1}$ 是 $\partial N$ 在 $S^{n+k-1} \times 0$ 中的护张, 且 $g_{1}^{-1}\left(S^{*+k-1} \times 1\right)=$ $\partial M, g_{1}^{-1}\left(S^{++k-1} \times 0\right)=\partial N$, 利用 $g_{1}$ 及 $N \subset^{n} D^{n+k}$, 则存在 $N \bigcup_{18 N} P$ 到 $D^{a+k} \bigcup_{1,++k-1 \times 0}$ $S^{n+k-1} \times I=D^{-+k}$ 的嵌入 $g$.

令 $W_{1}=\left(N U_{1 O N} P\right) \times I$, 则 $g \times i d: W_{1} \rightarrow\left(D^{n+k} \cup_{1 s^{n+k-1} \times 0} S^{n+k-1} \times I\right) \times I$ 是侻入, 由 于 $W_{1}$ 可看作 $N$ 到 $\left(N U_{\mid a N} P\right) \times 1 U_{10 \mathbf{y}} \partial M \times I=N U_{10 N} P$ 的配边, 类似地 $\left(D^{n+k} U_{1 s^{n+k-1} \times 0}\right.$ $\left.S^{n+k-1} \times I\right) \times I$ 也可看作 $D^{*+k}$ 到 $\left(D^{*+k} \bigcup_{1 s^{n+k-1} \times 8} S^{n+k-1} \times I\right) \times 1=D^{n+k}$ 的平凡配边, 因此由 $g \times i d$ 我们有嵌入

$$
G_{1}: W_{1} \rightarrow D^{n+k} \times I
$$

满足 $\quad G_{1}^{-1}\left(D^{n+k} \times 0\right)=N, \quad G_{1}^{-1}\left(D^{n+k} \times 1\right)=N \bigcup_{1 \partial N} P, \quad G_{1}^{-1}\left(S^{n+k-1} \times I\right)=P, \quad$ 这里 $\partial\left(N \cup_{i \mathrm{aN}} P\right)=\partial M$.

设 $W_{2}$ 是通过在 $N U_{10 N} P$ 的内部贴附一系列指数 $\leqslant\left[\frac{n}{2}\right]+1$ 手柄所给出的 $N U_{\mid O N} P$ 到 $M$ 的配边, 因为 $2 k \geqslant n+3$, 由引理 1 则有嵌入

$$
G_{2}: W_{2} \rightarrow D^{n+k} \times I
$$

满足 $G_{2}^{-1}\left(D^{n+k} \times 0\right)=N \bigcup_{1 \partial N} P, G_{2}^{-1}\left(D^{n+k} \times 1\right)=M, G_{2}^{-1}\left(S^{n+k-1} \times I\right)=\partial M \times I$.

由于 $W=W_{1} \cup W_{2}$, 利用 $G_{1}$ 和 $G_{2}$, 因此我们有羙入

$$
G: W \rightarrow D^{n+k} \times 1
$$

满足 $G^{-1}\left(D^{n+k} \times 0\right)=N, G^{-1}\left(D^{n+k} \times 1\right)=M, G^{-1}\left(S^{n+k-1} \times I\right)=P$.

设嵌入 $G$ 的法丛为 $\nu^{k}\left(W, D^{*+k} \times I\right)$, 因此 $\left.d\right|_{N}:(N, \partial N) \rightarrow\left(V^{k}(M), V^{k}(\partial M)\right)$ 可 扩张为一个映射 $D: W \rightarrow V^{k}(M)$, 满足 $D(P) \subset V^{k}(\partial M) ; b_{1}:\left(\nu\left(N, D^{s+k}\right), \nu\left(\partial N, S^{4+k-1}\right)\right) \rightarrow$ $\left(V^{k}\left(\nu_{M}\right), V^{k}\left(\nu_{\partial M}\right)\right)$ 可扩张为 $D$ 上的一个丛映射 $B:\left(\nu\left(W, D^{*+k} \times I\right), v\left(P, S^{*+k-1} \times I\right)\right) \rightarrow$ $\left(V^{k}\left(\nu_{M}\right), N^{k}\left(\nu_{\partial M}\right)\right)$ ，于是我们有 Thom 复形之间的映射

$$
\text { tb: }\left(T \nu\left(W, D^{n+k} \times I\right), T \nu\left(P, S^{n+k-1} \times I\right)\right) \rightarrow\left(T V^{k}\left(\nu_{M}\right), T V^{k}\left(\nu_{\bar{\alpha}}\right)\right)
$$

把 $W$ 在 $D^{n+k} \times l$ 中的法丛的余撮合成一点, 我们就得到一个酜射 


$$
C:\left(D^{n+k}, S^{m+k-1}\right) \times I \rightarrow\left(T \nu\left(W, D^{*+k} \times I\right), T \nu\left(P, S^{n+k-1} \times I\right)\right),
$$

于是组合 $\left.(C \subset t B)\right|_{\left(D^{n+k}, s^{n+k-1}\right) \times 0}=d$, 如令 $f=\left.G\right|_{M}$, 由 $\phi$ 的定义, 显然 $d([f])=d$, 这说. 明 $\phi$ 是满的.

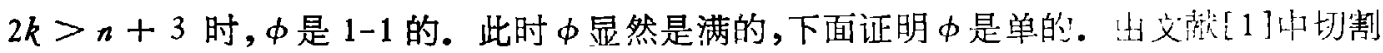
谈人引理和我们引理 1 , 因为在 $\phi$ 是满的证明中所贴附的手柄就同症而言走唯一的,因此 $\phi$ 是 单的.

$2 k \geqslant n+1$ 时, $\theta$ 是满的. 设 $q$ 充分大,例如 $q>n+1$, 利用同构 $\pi_{n+k}\left(Q^{q} \Sigma^{q} T V^{k}\left(\nu_{M}\right)\right.$, $\left.Q^{q} \Sigma^{q} T V^{k}\left(\nu_{\partial M}\right)\right) \cong \pi_{n+k+q}\left(\Sigma^{q} T V^{k}\left(\nu_{M}\right), \quad \Sigma^{q} T V^{k}\left(\nu_{\partial M}\right)\right) \cong \pi_{n+k+q}\left(T\left(V^{k}\left(\nu_{M}\right) \oplus \varepsilon^{q}\right), \quad T\left(V^{k}\right.\right.$ - $\left.\left.\left(\nu_{\partial M}\right) \oplus \varepsilon^{q}\right)\right)$, 因此对任元素 $d \in \pi_{n+k}\left(Q^{\infty} \Sigma^{\infty} T V^{k}\left(\nu_{M}\right), Q^{\infty} \Sigma^{\infty} T V^{k}\left(\nu_{\partial M}\right)\right) \cong \pi_{n+k}\left(Q^{q} \Sigma^{q} T V^{k}\right.$ $\left.\cdot\left(v_{M}\right), Q^{q} \Sigma^{q} T V^{k}\left(v_{\partial M}\right)\right)$, 不仿设 $d_{1} \in \pi_{n+k+q}\left(T\left(V^{k}\left(v_{M}\right) \oplus \varepsilon^{q}\right), T\left(V^{k}\left(\nu_{\partial M}\right) \oplus \varepsilon^{q}\right)\right)$ 是 $d$ 在上面 的同构下所对应的元素, 取 $d_{1}$ 的一个与 $V^{k}(M)$ 横截的表示, 我们仍记为 $d_{1}$, 于是 $N=d_{1}^{-1}$ $\cdot\left(V^{k}(M)\right)$ 是 $D^{n+k+q}$ 中的 $n$ 维整齐子流形, 目有 $\left.d_{1}\right|_{N}$ 上的丛映射 $b_{1}: \nu\left(N, D^{n+k+q}\right) \rightarrow V^{k}$ $\cdot\left(\nu_{M}\right) \oplus \varepsilon^{q}$ 。如 $d \in I_{*}^{-1}\left(C_{M}\right)$, 由文献 [8]中的 $(10.1)_{B}$, 则 $\left(\left.\pi \circ d_{1}\right|_{N}, b_{2} \circ b_{1}\right)$ 正规配边于一个平 凡的正规映射, 这里 $b_{2}: V^{k}\left(\nu_{M}\right) \oplus \varepsilon^{q} \rightarrow \nu_{M}^{k+q}$ 是 $\pi$ 上的丛映射. 于是存在三分量组 $\left(W^{n+1}\right.$, $F, B)$, 这里 $W$ 是连系 $N$ 和 $M$ 的配边, 由前面的命题我们可设 $W$ 是由 $N$ 通过贴附指数 $\leqslant\left[\frac{n}{2}\right]+$ 1 的手柄而得到, 因为 $q>n+1$, 我们可设 $W$ 整齐歕人在 $D^{n+k+q} \times I$ 中, 满足 $W \cap D^{n+k+q} \times$ $0=N, W \cap D^{n+k+q} \times 1=M, W \cap\left[S^{n+k+q-1} \times I\right]=\partial W-(\operatorname{int} M U \operatorname{int} N) ; F$ 是 $\left.\pi^{\circ} d_{1}\right|_{N} U$ $i d M$ 的扩张; $B$ 是 $F$ 上的丛映射且是 $b_{2} \circ b_{1} \cup i d \nu_{M}$ 的扩张.

因为 $O(k+q) / O(k)$ 是 $(k-1)$-连通的, 易知 $\pi: V^{k}(M) \rightarrow M$ 和 $\pi \mid v^{k}(\partial M): V^{k}(\partial M) \rightarrow$ $\partial M$ 均是 $k$-连通的, 又知 $2 k \geqslant n+1$ 且 $W$ 是由 $N$ 通过贴附一系列指数 $\leqslant\left[\frac{n}{2}\right]+1$ 的手柄 而得到,因此存在映射 $\tilde{F}: W \rightarrow V^{k}(M)$ 使下图可换

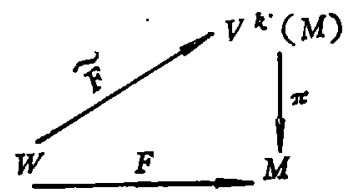

且 $\left.\tilde{F}\right|_{N}=\left.d_{1}\right|_{N}$, 这说明 $v\left(W, D^{*+k+q}\right)$ 可表示为 $k$ 维丛与 $q$ 维平凡丛的 Whitney 和, 由文 献 [9] 中定理 6.4, 说明 $W$ 可内漫于 $D^{*+k} \times 1$. 设此内浸对 $M$ 的限制为 $f$, 显然 $\theta(f)=d$.

$2 k>n+1$ 时， $\theta$ 是 1-1 的. 此时 $\theta$ 显然是满的, 下面我们说明 $\theta$ 是单的. 由于 $2 k>$ $n+1$, 在 $\theta$ 是满的证明中提升 $\widetilde{F}$ 就同伦而言是唯一的,因此 $\theta$ 是单的.

周学光教授向作者提供了文献 [1]且提出了许多指导性意见,作者表示衰心地感谢。

\section{参考献}

[ 1 ] Rigdon, R. \& Williams, B., Lecture Notes in Math., 657(1978), 423-453.

[2] Glover, H. \& Mislin, G., Proc. Amer. Math. Soc., 4:(1974), 443-448.

[3] Rees, E., Bull. London Math. Soc, 3(1971), 356--362.

[4] Salomonsen, H., On the existence and classification of differentiable embeddings in the metastable range, Aar- 
hus Notes, 1973

[5] Williams. B., Pacilic J. Math.. 84(1979), 217-224.

[6] Rigcion, R., J. L. M. S, 11(1975), 233-244.

[ 7 ] Browder, W., Surgery of Simply-connected Manifolds, Springer-Verlag, 1972.

[8] Wall, C. T. C., Surgery of Compact Manijolds, Aca emic Press, London and New York, 1970.

[ 9 ] Hirsch, M. W., Trans. Amer. Math. Soc, 93(1959), 242-276. 